\section{Obstetric Update: Antepartum Assess- ment of the Foetus and its Environment}

Frank A. Manning, MD
In the past decade there has been a steady and dramatic fall in perinatal mortality rates (PNM) such that most centres now report rates of less than 12 per 1000 . Most of the decrease in PNM has been the result of improved methods of neonatal care, whereas stillbirth rates have not decreased proportionately. Currently there is intense research into methods for detecting the foetus at risk of death or damage in utero. As a result, conventional indirect endocrinological methods for detecting the foetus at risk (estriols, human placental lactogen) have been replaced with more sensitive direct foetal biophysical measures such as antepartum foetal heart rate monitoring and composite foetal biophysical activity assessment. Dynamic ultrasound methods (Doppler and real time B-mode scan) have become the standard for foetal assessment. Application of these methods in a high risk population have reduced stillbirth rates to as low as three per 1000 , a four-fold reduction from expected stillbirth rates in an untested population (14 per 1000).

\section{Antepartum Foetal Assessment}

A. Antepartum foetal Heart Rate Testing

Two methods for foetal evaluation based on antepartum foetal heart rate recording are described. The first, termed a non-stress test (NST) is based upon a foetal biophysical coupling of heart rate acceleration with foetal movement. The normal (reactive) test result requires the presence of at least two accelerations of $\geq 15 \mathrm{~b}$.p.m. and $\geq 15$ seconds duration in a 20-minute period. A test that fails to become reactive in two consecutive 20 minute periods ( $40 \mathrm{~min}$ ) ) is termed as non-reactive (abnormal). Foetal death with a week of a reactive NST occurs at a rate eight per 1000 and at a rate of 33 per 1000 with a non-reactive test. ${ }^{1}$ Major disadvantages of this method include a high false positive rate (NR-NST), an unacceptably high false negative rate
(R-NST), and inability to detect major congenital anomalies. Nonetheless, because of the ease with which the test is done and the widespread availability of Doppler foetal heart rate monitors, NST testing has become the most common method for foetal assessment.

The second method, termed an oxytocin challenge test or contraction stress test (CST) is based on the presence or absence of late decelerations of the foetal heart rate with induced or spontaneous uterine contractions. ${ }^{2}$ The absence of late decelerations (negative CST) is associated with a foetal death rate of 24 per 1000 whereas the presence of repetitive late decelerations (positive CST) carries a foetal death rate of at least 125 per 1000 . This test is somewhat less sensitive than the NST and frequently requires maternal oxytocin infusion with its attending risks. In most centres, the NST has replaced the CST and the latter is only rarely used as an ancillary test in the immature foetus.

B. Composite Foetal Biophysical Profile Scoring The advent of high resolution dynamic B-mode ultrasound imaging methods now permit detailed assessment of several foetal biophysical activities including foetal breathing, gross body movement, organized purposeful and reflex movements (hand to mouth, sucking, eye movement), foetal flexor/

From the Diyision of Maternal-Fetal Medicine, Department of Obstetrics, Gynecology, and Reproductive Sciences, Health Sciences Centre and the University of Manitoba.

Address reprint requests for Perinatal Medicine Section (Complete) to Department of Anesthesia, University of Manitoba, Lennox Bell 315, 700 William Avenue, Winnipeg, Manitoba R3E 023. 
extensor tone and response to intrinsic and extrinsic stimuli. Present methods permit nearly as detailed an examination of the foetus as is possible in the neonate. The intrauterine cnvironment, as refected by amniotic fluid volume and placental position and morphology is also readily determined with these dynamic ultrasound methods. The foetal biopliysical profile scoring, based on assessment of five discrete variables (foetal movement, tone, breathing, reactivity, and amniotic fluid volume), is a highly specific method for differentiating the normal from the compromised foetus. ${ }^{3}$ When all variables are present and normal, foetal death occurs at a rate of less than one per 1000 whereas when all variables are abnormal, foetal death in untreated foetuses occurs at a rate of 400 per 1000 . Application of this method to a high risk population has reduced perinatal mortality in structurally normal foetuses to less than four per $1000 .^{4}$

\section{Antepartum Detection of Foetal Anomalies}

In the untested population approximately 40 per cent of stillbirths are a result of chronic asphyxia while 20-25 per cent of deaths are due to congenital anomalies. A dramatic fall in asphyxial death rates produced by foetal biophysical variable monitoring has resulted in a relative increase in the number of deaths due to anomaly, such that in populations screened by foetal biophysical profile scoring methods, in excess of 80 per cent of deaths are due to anomaly. Fortunately, the introduction of dynamic ultrasound methods in perinatal medicine has resulted in improved antepartum detection of structural anomalies such that now in excess of 75 of all anomalous foetuses may be recognized before delivery. ${ }^{5}$ Anomaly diagnosis is based on detection of both structural and functional defects, e.g., failure to visualize kidneys or bladder and failure to produce urine in response to maternal furosemide injection in foetuses with renal agenesis (Potter's Syndrome). Antepartum detection of a major anomaly can have a profound impact on subsequent pregnancy management. Detection of a lethal nontreatable anomaly (c.g. anencephaly) can prevent operative intervention for foetal distress. This point is lesis than theoretical since upwards of 0.5 per cent of all caesarean sections are done for foetuses with a lethal anomaly.* In contrast, some anomalies are

*Monis M, Manning FA, Unpublished Observation, 1982. amenable to treatment. In the mature foetus with disorders such as omphalocoele, intestinal obstruction, supraventricular tachyarrythmia and congestive heart failure, controlled delivery and immediate neonatal treatment can be life-saving. In the very immature foetus intrauterine surgical treatment may be used to prolong survival to a point where extrauterine survival is possible. Such circumstances include chronic vesicoamniotic shunting in foetuses with posterior urethral valves and ventriculoamniotic shunting in foetuses with progresșive hydrocephalus.

\section{Assessment of Foetal Growth}

Intrauterine growth retardation is associated with a six- to eight-fold increase in perinatal mortality. Developing highly accurate ultra-sound methods for determining foetal age, weight and growth now permit detection of most foetuses with intrauterine growth retardation. Foetal age is determined by ultrasound methods such as foetal biparietal diameter, femur length and abdominal circumference. When used in combination, before 30 weeks gestation, foetal age may be determined accurately to within \pm seven days. ${ }^{6}$ Foetal weight estimates derived from abdominal girth or from composite foetal volumetric estimates are accurate to within \pm 8 per cent. ${ }^{7}$ Thus it is now possible to construct weight-to-age foetal curves to monitor foetal growth. Such methods permit detection of the foetus with intrauterine weight loss. By combination of these methods with congenital anomaly screening and qualitative amniotic fluid volume determination it is now possible not only to detect almost all growth retarded foetuses but to also determine the aetiology of the disorder (normal small infant, dysmaturity syndrome, congenital anomaly). Perinatal mortality in pregnancies complicated by intrauterine growth retardation can be reduced from $>50 / 1000$ to $<10 / 1000$ with these methods. ${ }^{8}$

\section{E. Other Considerations}

The effect of dynamic ultrasound methods on the practice of high risk obstetrics is remarkable. Multiple pregnancy detection now approaches 100 per cent. Maternal and foetal complications associated with amniocentesis have fallen significantly. ${ }^{9}$ As a result more liberal use of amniocentesis has produced a decrease in iatrogenic premature deliv- 
ery, increased amniotic fluid culture and karyotyping for genetic disorders, and better recognition and treatment of the foetus at risk for intrauterine sepsis (e.g., premature rupture of membranes). Antepartum assessment and treatment of the Rh isoimmunized foetus have improved such that survival of the severely non-hydropic isoimmunized foetus now approaches $100 \%$ and traumatic death during intrauterine transfusion has virtually disappeared. ${ }^{10}$

\section{Conclusion}

Until recently the practice of high-risk obstetrics has been limited because of the inability to assess the perinate directly. The application of dynamic ultrasound now enables both structural and functional assessment of the foetus and its environment. Thus it is now possible to begin to transfer the principles of physical diagnosis used in extrauterine medicine to the foetus in utero. This major advance has resulted in a four- to six-fold decrease in foetal death rate, a detection of anomalies approaching 80 per cent, as well creating opportunities to reduce maternal and foetal morbidity associated with anomalies, and detection of almost all growth retarded foetuses and twins. Technical procedures have been greatly facilitated. Despite these advances, however, it is likely that the greatest positive influence of the technique will be the recognition and management of the foetus as a separate patient.

\section{References}

1 Evertson LR, Gauthier RI, Schifrin BS et al. Antepartum Fetal Heart Rate Testing. I. Evolution of the non-stress test. Am J Obstet Gynecol 1979; 133: 29-34.

2 Ray $M$, Freeman $R K$, Pine $S$. Clinical experience with the Oxytocin Challenge Test. Am J Obstet Gynecol 1972; 114: 1-8.

3 Manning FA, Platt LD, Sipos L. Antepartum Fetal Evaluation: Development of a Fetal Biophysical Profile Score. Am J Obstet Gynecol 1980; 136: 787-94.

4 Manning FA, Basket TF, Morrison l, et al. Fetal Biophysical Profile Scoring: A prospective study of 1184 high risk patients. Am J Obstet Gynecol 1981; 140: 289-94.

5 Manning FA, Morrison I, Lange IR et al. Antepartum Determination of Fetal Health: Composite Fetal Biophysical Profile Scoring. Clin Perinat 1982; 9: 285-96.

6 Sabbagha $R F$. IUGR: Antenatal Diagnosis by Ultrasound. Obstet Gynecol 1978; 52: 252-7.

7 Manning FA, Thompson T, Khitrov Get al. Antepartum calculation of fetal weight by ultrasound: $A$ Comparative Study. Proc Sac Obstet Gynecol Can June, 1982 (Abstract).

8 Morrison I, Manning FA, Lange IR. Antepartum detection of IUGR: Prospective study of 1000 referred cases. In preparation, 1982.

9 Platt $L D$, Manning FA, LeMay M. Real Time B-scan Directed Amniocentesis. Am I Obstet Gynecol 1978; 130: 700-4.

10 Harman CR, Manning FA, Bowman JM et al. Severe Rh Disease: Poor outcome is not incvitable. Am J Obstet Gynecol (In press) 1982. 